\title{
Estudos Quantitativos em Mamografia Digital Contrastada de Dupla Energia
}

\author{
F. V. Dumaszak*, D. M. Cunha* \\ *Instituto de Física, Universidade Federal de Uberlândia, 38400-902 Uberlândia, Minas Gerais, Brazil \\ e-mail: fabiodumaszak@live.com
}

Resumo: A detecção de massas suspeitas em exames mamográficos é comprometida pela superposição de tecido mamário na imagem. A técnica de mamografia digital de dupla energia utiliza duas exposições com energias diferentes obtendo assim duas imagens mamográficas, onde a subtração dessas cancelaria parte do tecido sobreposto na imagem melhorando a visualização. $\mathrm{O}$ desenvolvimento de carcinomas induz angiogênese, ou seja, um aumento na vascularização na região da lesão. Logo, com adição de uma solução de Iodo intravenosa esta se acumularia na região do carcinoma gerando um objeto de contraste que pode ser destacado pelo uso da técnica de dupla energia.

Neste trabalho, calculamos os sinais obtidos no detector $\left(S_{l}, S_{h} \dot{i}\right.$ referente as duas exposições podemos obter uma função inversa que calcula a espessura mássica de Iodo em função do sinal obtido, fornecendo assim dados quantitativos a respeito da concentração de Iodo. Comparando os valores obtidos por dados de calibração para a espessura mássica de Iodo e os valores obtidos através da função inversa verificamos que estes são iguais, mostrando que a função se adequa ao modelo proposto para mamografia digital contrastada de dupla energia.

Palavras-chave: Mamografia, Dupla Energia, Função Inversa.

\begin{abstract}
The detection of suspicious masses in mammographic exams are compromised by breast tissue overlapping on the image. The dual energy digital mammography technic uses two exposures with different energies obtaining two mammographic imagens, where their subtraction would cancel part of the overlapping tissue enhancing its visualization. The developing of carcinomas induces angiogenesis, an increase on the vascularization in the region of the lesion. Therefore, with the addition of an intravenous Iodine solution it would accumulate at the carcinoma region generating a contrasted object that would be highlighted by the dual energy technique.

In this work we calculated the signals obtained by the detector $\left(S_{l}, S_{h} \dot{i}\right.$ from the two exposures we can fit an inverse function that calculate the mass thickness of
\end{abstract}

Iodine as a function of the signal, providing quantitative data regarded to the Iodine concentration. Comparing

the values obtained by calibration data for the mass thickness of Iodine and those obtained from the inverse function we verify that they are equal, showing that the function fits the proposed model for contrasted dual energy digital mammography.

Keywords: Mammography, Dual Energy, Inverse Function.

\section{Introdução}

A detecção precoce do câncer de mama é um fator de grande benefício no tratamento dessas lesões e a mamografia é a principal técnica empregada para o diagnóstico[1], o qual consiste na detecção de microcalcificações ou massas de tecido anormais. Entretanto a visualização dessas é comprometida pela sobreposição de tecido mamário na imagem.

Uma alternativa para esse problema é a técnica de mamografia digital de dupla energia que consiste no uso de duas exposições consecutivas em um curto espaço de tempo onde duas imagens são obtidas, uma para cada exposição. A subtração dessas duas imagens cancelaria maior parte dos tecidos sobrepostos na imagem[2].

$\mathrm{O}$ desenvolvimento de carcinomas induz angiogênese, ou seja, um aumento na vascularização na região da lesão[3]. Na introdução de um contraste de iodo intravenoso na mama, essas regiões obtêm maior acúmulo do contraste devido a supervascularização, tornando-o mais visível à mamografia. Os artigos publicados por Dromain[4,5] mostraram bons resultados usando um contraste de Iodo em mamografia de dupla energia.

Neste trabalho foram simulados os sinais obtidos na mamogragia digital de dupla energia em função da porcentagem de tecido adiposo e glandular na mama e da espessura mássica de Iodo. Assim, podemos encontrar uma função inversa que possibilita calcular a quantidade de Iodo através do sinal obtido no detector, com o objetivo de obter informações quantitativas a respeito da concentração de Iodo. 


\section{Materiais e métodos}

\section{Modelo Geométrico}

O modelo geométrico adotado consiste em um tubo de raios $\mathrm{X}$ com anodo de Tungstênio(W). O feixe é filtrado por uma janela de berílio(Be) de $0,5 \mathrm{~mm}$ na saída do tubo e mais uma filtração adicional de $0,3 \mathrm{~mm}$ de $\operatorname{cobre}(\mathrm{Cu})$, que tem boa eficiência na redução de dose glandular em exames mamográficos [6].

$\mathrm{O}$ feixe de raios $\mathrm{X}$ encontra em seu caminho uma bandeja de compressão de espessura $3 \mathrm{~mm}$, a mama comprimida, um suporte para a mama de $1,5 \mathrm{~mm}$, uma grade anti-espalhamento, e o detector. O suporte da mama e a bandeja de compressão são compostos de polimetilmetacrilato (PMMA). A grade anti-espalhamento é colocada entre o suporte da mama e o detector para barrar parte da radiação espalhada. A grade é linear e possui paredes de chumbo de $0,016 \mathrm{~mm}$ de largura, entrespaço de PMMA de largura $0,3 \mathrm{~mm}$ e altura da grade de $1,5 \mathrm{~mm}[7]$.

A mama foi modelada como uma composição homogênea de tecido glandular, tecido adiposo e um objeto contrastante de Iodo, tendo expessura total $T$. A densidade da mama depende de sua glandularidade, ou seja, porcentagem de tecido glandular e adiposo, sendo calculado segundo o modelo de Boone[8].

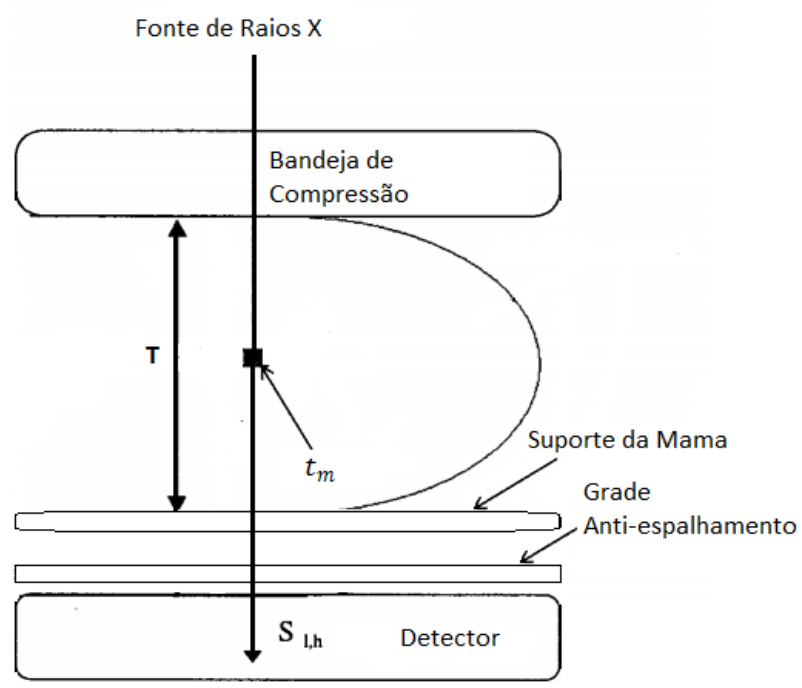

Figura 1:Modelo geometrico utilizado adaptado de Lemacks [8].

\section{Sinal do detector}

O sinal obtido no detector é calculado através atenuação dos materiais no caminho do feixe de raio $\mathrm{X} \mathrm{e}$ da resposta do detector. Usando o modelo proposto por Lemacks e assumindo um feixe polienergético temos que o sinal é dado pela equação[9]:

$$
\begin{array}{r}
S_{j}=\int d E \times R_{j} \times d^{2} \times \Phi_{j}(E) \times \\
\times e^{-\left(\mu_{\text {mama }} \cdot\left(T-\frac{t_{m}}{\rho_{I}}\right)+\frac{\mu_{I}}{\rho_{I}} \cdot t_{m}\right)} \times A(E) \times Q(E)
\end{array}
$$

Onde $T$ é a expessura da mama, $\rho_{I}$ a densidade do Iodo e $t_{m}$ a expessura mássica de Iodo no tecido. O indice $j=l, h$, é referente ao espectro usado, de alta $(h)$ ou baixa $(l)$ energia. $R_{j}(C / K g)$ são as exposições não atenuadas de alta e baixa energias no plano do detector, $d(\mathrm{~cm})$ é o tamano do pixel, $\Phi_{j}(E)\left(\right.$ fótons $\left./\left(\mathrm{cm}^{2} \cdot \mathrm{keV} . \mathrm{C} / \mathrm{kg}\right)\right)$ é a distribuição da fluência de alta e baixa energia por unidade de energia e de exposição na chegada ao detector, $A(E)$ é a fração de absorção dos fótons que chega ao detector em função da energia $E(k e V) e \quad Q(E)$ é a função de resposta ou ganho do detector, ou seja, a quantidade de sinal gerado pela energia que chega ao detector. Neste trabalho consideramos um detector de Iodeto de Césio (CsI).

Os espectros usados para calcular o sinal no detector foram obtidos através de um aplicativo gerador de dados de spectro (SpekCalc)[10]. Para realizar a simulação empregamos espectros gerados por tensões $28 \mathrm{kVp}$ e $46 \mathrm{kVp}$; possibilitando diferenciar a absorção fotoelétrica da camada $\mathrm{K}$ do Iodo em 33,12keV[11].

Análogo à densidade optica, a densidade de raios $\mathrm{x}$ $D_{j} \quad$ é dada pela função logarítmica[8]:

$$
D_{j}=\ln \left(\frac{S_{j}^{a}}{S_{j}}\right) j=l, h
$$

O sinal de referência $S_{j}^{a} \quad$ é obtido da mesma forma que o sinal $S_{j}$ porém, considerando a mama como tecido $100 \%$ adiposo na equação (2).

A função logarítmica $D_{j}$ representa a atenuação média do feixe por raio $\mathrm{X}$ incidente e também a resposta do detector, considerando o tecido mamário como uma mistura homogênea de tecido adiposo, glandular e o objeto de contraste de iodo. Logo, temos que o sinal $D_{j} \quad$ está em função de $S_{j}^{a}$ e $S_{j}$, sendo uma função dependente da glandularidade da mama(porcentagem de tecido glandular) e da espessura mássica de Iodo $\left(\mathrm{mg} / \mathrm{cm}^{2}\right)$ presente no tecido. 
Obtendo-se o sinal no detector em função da espessura mássica $D_{j}\left(t_{m}\right)$, podemos calcular funções inversas que aproximam o valores de $t_{m}$ em função dos sinais

$D_{l}$ e $D_{h}$. O uso de feixes poli-energéticos em imagens diagnósticas e a dependência da energia na atenuação do feixe resultam em uma dependência não linear entre $\left[D_{l}, D_{h}\right]$ e $\left[t_{m}\right]$. A função polinomial que mais se ajusta a essa dependência é uma função cúbica do tipo[12]:

$$
t_{m}=c_{0}+c_{1} D_{l}+c_{2} D_{h}+c_{3} D_{l}^{2}+c_{4} D_{h}^{2}+c_{5} D_{l} D_{h}(3)+
$$

Onde $C_{i}$ são os coeficientes do polinômio, predeterminados por dados de calibração. Logo, obtendo os valores de $C_{i}$ temos uma função que permite calcular os valores de $t_{m}$ através dos sinais obtidos na mamografia digital.

\section{Resultados}

Considerando uma mama com $30 \%$ de tecido glandular calculamos os coeficientes da função polinomial para mamas com espessuras $T$ de 2, 4, 6 e $8 \mathrm{~cm}$ que estão dispostos na tabela 1 .

Tabela 1:Coeficientes da função cúbica para mamas com $30 \%$ de tecido glandular de expessuras 2, 4, 6 e $8 \mathrm{~cm}$.

\begin{tabular}{cccc}
\hline \multicolumn{4}{c}{$T(\mathrm{~cm})$} \\
2 & 4 & 6 & 8 \\
\hline $2.1 \times 10^{-3}$ & $2.1 \times 10^{-3}$ & $2.1 \times 10^{-3}$ & $2.1 \times 10^{-}$ \\
5.5 & 9.1 & 3.9 & 1.4 \\
$\times 10^{-4}$ & $\times 10^{-4}$ & $\times 10^{-4}$ & $\times 10^{-5}$ \\
6.3 & 2.7 & 7.9 & 1.2 \\
$\times 10^{-4}$ & $\times 10^{-4}$ & $\times 10^{-4}$ & $\times 10^{-3}$ \\
-6.7 & -2.9 & -1.6 & -2.8 \\
$\times 10^{-6}$ & $\times 10^{-6}$ & $\times 10^{-5}$ & $\times 10^{-5}$ \\
1.3 & 5.9 & 2.6 & 4.1 \\
$\times 10^{-5}$ & $\times 10^{-6}$ & $\times 10^{-5}$ & $\times 10^{-5}$ \\
-5.0 & -2.5 & -8.9 & -1.1 \\
$\times 10^{-6}$ & $\times 10^{-6}$ & $\times 10^{-6}$ & $\times 10^{-5}$ \\
6.4 & 2.9 & 1.7 & 3.5 \\
$\times 10^{-9}$ & $\times 10^{-9}$ & $\times 10^{-9}$ & $\times 10^{-8}$ \\
-3.4 & -1.6 & -8.7 & -1.6 \\
$\times 10^{-9}$ & $\times 10^{-9}$ & $\times 10^{-9}$ & $\times 10^{-8}$ \\
\hline
\end{tabular}

Usando a função polinomial obtida para a mama $30 \%$ glandular e espessura $T$ igual a $4 \mathrm{~cm}$ calculamos alguns valores de espessura mássica $t_{m}$ e comparamos com valores verdadeiros usados na simulação. Obtendo assim o gráfico da figura 2.

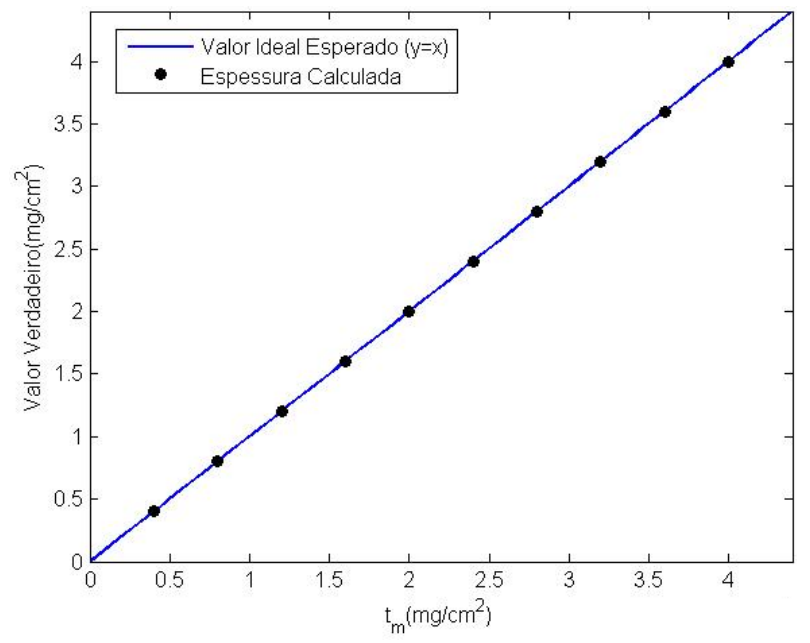

Figura 2: Valores verdadeiros de espessura mássica de Iodo contra os valores calculados pela função inversa para uma mama com $30 \%$ de tecido glandular e $4 \mathrm{~cm}$ de espessura.

\section{Discussão}

Considerando uma mama com $30 \%$ de tecido glandular e $70 \%$ de tecido adiposo, calculamos os coeficientes de algumas funções para mamas com espessuras de 2, 4, 6 e $8 \mathrm{~cm}$ mostrados na tabela 1. Para saber se os valores de coeficientes geram uma função que se adequa aos dados obtidos calculamos o erro médio quadrático(RMSE) para todos os casos. Os valores de RMSE variam de 0 a 1 , sendo que quanto mais próximo de 1 melhor a curva representa os dados fornecidos. Para todos os casos calculados, obtivemos RMSE igual a 1, mostrando que a curva se ajusta bem aos dados fornecidos.

Comparando os valores obtidos por dados de calibração para a espessura mássica de Iodo e os dados obtidos através da função inversa vemos que estes são iguais. Como vemos na figura 2 , os valores verdadeiros de

$t_{m}$ plotados contra os valores obtidos pelo polinômio para os mesmos sinais [ $\left.\begin{array}{lll}D_{l} & D_{h}\end{array}\right]$ coincidem sobre uma reta do tipo $\mathrm{y}=\mathrm{x}$. Mostrando assim que a função polinomial proposta se adequada para a modelagem desse problema.

\section{Conclusão}

Através dos resultados obtidos temos que o modelo proposto para gerar uma função inversa, do tipo polinômio de terceiro grau, permite calcular dados quantitativos na mamografia digital de dupla energia. Também vimos que o modelo de função proposto se adequa ao caso da mamografia contrastada, permitindo calcular a quantidade de Iodo na imagem fornecendo dados quantitativos sobre a imagem. Logo concluimos que 
essa técnica tem potencial para trazer uma possível melhoria na detecção e análise de massas suspeitas na mama. Entretanto, mais estudos ainda são necessários a fim de investigar a influência da flutuação estatística e da radiação espalhada na determinação dos coeficientes da função.

\section{Agradecimentos}

Gostaríamos de agradecer à Fundação de Amparo à Pesquisa de Minas Gerais(FAPEMIG) pela bolsa de iniciação científica concedida, a qual incentivou a realização deste trabalho.

\section{Referências}

[1] K. Smigel, "Breast cancer death rates decline for white w o m e n, , J . N a t 1 . Cancer Inst. $\sim 1940-1978$ ! 87, $173 \sim 1995$.

[2] P. C. Johns and M. J. Yaffe, "Theoretical optimization of dual-energy

X-ray imaging with applications to mammography," Med. $\mathrm{Phys}$. 12 , $289-$ $296 \sim 1985$ !.

[3] Weidner N, Semple JP, Welch WR, et al. Tumor angiogenesis and metastasis: correlation in invasive breast carcinoma. N Engl J M ed 1991; 324:1-8

[4] Dromain C, Balleyguier C, Muller S, Mathieu MC, Rochard F, Opolon Sigal R: Evaluation of tumor angiogenesis of breast c a r c i n o m a u s ing contrast enhanced digital mammography. Am J Roentgenol $2006, \quad 187$ : W528-W537.

[5] Dromain C, Thibault F, Muller S, Rimareix F, Delaloge S, T a r d i o n A, Balleyguier C. Dual-energy contrast-enhanced digital mammography: initial clinical results. Eur Radiol 2011, 21:565-574

[6] Ullman G, Sandborg M, Dance D. R, Yaffe M and Carlsson G A. A search for optimal x-ray spectra in i o d i n e c o n t r a s t media mammography. Phys. Med. Biol. 50 (2005) 31433152

[7] Rezentes P S, Almeida A, Barnes G T. Mammography Grid Performance1. Radiology 1999; 210:227-232

[8] Boone J M. Glandular breast dose for monoenergetic and high-energy X-ray beams: Monte Carlo assessment. Radiology 1999; 213:23-37.

[9] Lemacks M R, Kappadath S C, Shaw C C, Liu X, and Whitman G J. A dual-energy subtraction technique for $\mathrm{microcalcification} \quad$ i m a $\mathrm{ging}$ in digital mammography-A signal-to-noise analysis. Medical Physics 2002, Vol. 29, No. 8.
[10] Poludniowski G1, Landry G, DeBlois F, Evans PM, Verhaegen F. SpekCalc: a program to calculate photon spectra from tungsten anode x-ray tubes. Phys Med Biol. 2009 Oct 7;54(19):N433-8.

[11] Berger, M.J., Hubbell, J.H., Seltzer, S.M., Chang, J., Coursey, J.S., Sukumar, R., Zucker, D.S., and Olsen, K. (2010), XCOM: Photon Cross Section Database (version 1.5). [ O n lin e ] Ava i lable: http://physics.nist.gov/xcom [Saturday, 06-Aug-2016 15:28:02 EDT]. National Institute of Standards and Technology, Gaithersburg, MD.

[12] Kappadath S C and Shaw C C. Quantitative evaluation of dual-energy digital mammography for calcification imaging. Phys. Med. Biol. 49 (2004) 2563-2576 